\title{
Acyclovir-Resistant Cutaneous Herpes Simplex Virus in DOCK8 Deficiency
}

\author{
Christina R. Hopkins, MD; Nicholas Lowe, MD; Grace Lee, MD
}

\section{PRACTICE POINTS}

- Patients with dedicator of cytokinesis 8 (DOCK8) deficiency are susceptible to development of severe recalcitrant viral cutaneous infections, including herpes simplex virus (HSV).

- Dermatologists should be aware that prophylactic acyclovir may not be sufficient for HSV suppression in the setting of severe immunodeficiency.

- Viral culture should be performed on suspicious lesions in DOCK8-deficient patients despite acyclovir prophylaxis, and the threshold for HSV resistance testing should be low.

- Acyclovir-resistant cutaneous HSV lesions require escalation of therapy, which may include addition of foscarnet, cidofovir, or subcutaneous pegylated interferon alfa-2b to the therapeutic regimen.

Patients with dedicator of cytokinesis 8 (DOCK8) deficiency are susceptible to development of severe viral cutaneous infections, including herpes simplex virus (HSV). We report a 32-month-old girl with homozygous $D O C K 8$ deficiency who developed a posterior auricular cutaneous lesion that was culture positive for HSV despite acyclovir prophylaxis. Resolution of this tesion was only observed after addition of foscarnet to the treatment regimen. Prophylactic acyclovir may be insufficient for suppression of cutaneous HSV in patients with DOCK8 deficiency, and a high index of suspicion for viral resistance is necessary for prompt initiation of appropriate antiviral treatment in these patients.

Cutis. 2021;108:218-220.

$\square$ edicator of cytokinesis 8 (DOCK8) deficiency is the major cause of autosomal-recessive hyper-IgEsyndrome. ${ }^{1}$ Characteristic clinical features including eosinophilia, eczema, and recurrent
Staphylococcus aureus cutaneous and respiratory tract infections are common in DOCK 8 deficiency, similar to the autosomal-dominant form of hyper-IgE syndrome that is due to deficiency of signal transducer and activation of transcription 3 (STAT-3). ${ }^{1}$ In addition, patients with DOCK8 deficiency are particularly susceptible to asthma; food allergies; lymphomas; and severe cutaneous viral infections, including herpes simplex virus (HSV), molluscum contagiosum, varicella-zoster virus, and human papillomavirus. Since the discovery of the DOCK8 gene in 2009, various studies have sought to elucidate the mechanistic contribution of DOCK8 to the dermatologic immune enyironment. ${ }^{2}$ Although cutaneous viral infections such as those caused by HSV typically are short lived and selflimiting in immunocompetent hosts, they have proven to be severe and recalcitrant in the setting of DOCK 8 deficiency. ${ }^{1}$ Herein, we report the case of a 32-month-old girl with homozygous DOCK8 deficiency who developed acyclovir-resistant cutaneous HSV.

\section{Case Report}

A 32-month-old girl presented with an approximately 2 -cm linear erosion along the left posterior auricular sulcus at month 9 of a hospital stay for recurrent infections. Her medical history was notable for multiple upper respiratory tract infections, diffuse eczema, and food allergies. She had presented to an outside hospital at 14 months of age with herpetic gingivostomatitis and eczema herpeticum that was successfully treated with acyclovir. She was readmitted at 20 months of age due to Pneumocystis jiroveci pneumonia, pancytopenia, and disseminated histoplasmosis. Prophylactic oral acyclovir (20 mg/kg twice daily) was started, given her history of HSV infection. Because of recurrent infections, she underwent an immunodeficiency workup. Whole exome sequencing analysis revealed a homozygous deletion c. $\left(528+1 \_529-1\right) \_\left(1516+1 \_1517-1\right)$ del in DOCK8

\footnotetext{
Dr. Hopkins is from the Department of Dermatology, Baylor College of Medicine, Houston, Texas. Dr. Lowe is from the Department of Dermatology, Intermountain Medical Group, Salt Lake City, Utah. Dr. Lee is from the Department of Pediatric Dermatology, Texas Children's Hospital, Houston. The authors report no conflict of interest.

The eTable is available in the Appendix online at www.mdedge.com/dermatology.

Correspondence: Grace Lee, MD, Texas Children's Hospital, Department of Pediatric Dermatology, 6701 Fannin St, Mark Wallace Tower, 8th Floor, Houston, TX 77030 (gllee@texaschildrens.org).

doi: 10.12788 /cutis.0364
} 
gene-affecting exons 5 to 13 . The patient was transferred to our hospital for continued care and as a potential candidate for bone marrow transplant following resolution of the disseminated histoplasmosis infection.

During her hospitalization at the current presentation, she was noted to have a 2 -cm linear erosion along the left posterior auricular sulcus. Initial wound care with bacitracin ointment was applied to the area while specimens were obtained and empiric oral acyclovir therapy was initiated $(20 \mathrm{mg} / \mathrm{kg} 4$ times daily [QID]), given a clinical impression consistent with cutaneous HSV infection despite acyclovir prophylaxis. Direct immunofluorescence and viral cultures were positive for HSV-1, while bacterial cultures grew methicillin-susceptible $S$ aureus. Cephalexin and mupirocin ointment were started, and acyclovir was continued. After 2 weeks of therapy, there was no visible change in the wound; cultures were repeated, again showing the wound contained HSV. Bacterial cultures this time grew Pseudomonas putida, and the antibiotic regimen was transitioned to cefepime.

After no response to the continued course of therapeutic acyclovir, HSV cultures were sent to the Centers for Disease Control and Prevention for resistance testing, and biopsy of the lesion was performed by the otolaryngology service to rule out malignancy and potential alternative diagnoses. Histopathology showed only reactive inflammation without visible microorganisms on tissue HSV-1/HSV-2 immunostain; however, tissue viral culture was positive for HSV-1. The patient was transitioned back to acyclovir (intravenous [IV] $20 \mathrm{mg} / \mathrm{kg}$ QID) with the addition of empiric foscarnet (IV $40 \mathrm{mg} / \mathrm{kg} 3$ times daily) given the worsening appearance of the lesion. The HSV acyclovir resistance test results from the Centers for Disease Control and Prevention returned soon after and were positive for resistance (median infectious dose, $3.29 \mu \mathrm{g} / \mathrm{L}$ [reference interval, sensitive $<2.00 \mu \mathrm{g} / \mathrm{L}$; resistant $>1.90 \mu \mathrm{g} / \mathrm{L}]$ ). The patient eompleted a 21 -day course of combination foscarnet and acyclovir therapy, during which time the lesion showed notable improvement and healing. The patient was continued on prophylactic acyclovir (IV $20 \mathrm{mg} / \mathrm{kg}$ QID). Unfortunately, the patient eventually died due to complications related to pneumonia.

\section{Comment}

Infection in Patients With DOCK8 Deficiency-The gene DOCK8 has emerged as playing a central role in both innate and adaptive immunity, as it is expressed primarily in immune cells and serves as a mediator of numerous processes, including immune synapse formation, cell signaling and trafficking, antibody and cytokine production, and lymphocyte memory. ${ }^{3}$ Cells that are critical for combating cutaneous viral infections, including skin-resident memory $\mathrm{T}$ cells and natural killer cells, are defective, which leads to a severely immunocompromised state in DOCK8-deficient patients with a particular susceptibility to infectious and inflammatory dermatologic disease. ${ }^{4}$
Herpes simplex virus infection commonly is seen in DOCK8 deficiency, with retrospective analysis of a DOCK8-deficient cohort revealing HSV infection in approximately $38 \%$ of patients. ${ }^{5}$ Prophylactic acyclovir is essential for DOCK8-deficient individuals with a history of HSV infection given the tendency of the virus to reactivate. ${ }^{6}$ However, despite prophylaxis, our patient developed an HSV-positive posterior auricular erosion that continued to progress even after increase of the acyclovir dose. Acyclovir resistance testing of the HSV isolated from the wound was positive, confirming the clinical suspicion of the presence of acyclovir-resistant HSV infection.

Acyclovir-Resistant HSV-Acyclovir-resistant HSV in immunosuppressed individuals was first noted in 1982, and most cases since then, have occurred in the setting of AIDS and in organ transplant recipients. ${ }^{6}$ Few reports of acyclovir-resistant HSV in DOCK8 deficiency exist, and to our knowledge, our patient is the youngest DOCK8-deficient individual to be documented with acyclovir-resistant HSV infection. ${ }^{1,7-15}$ We identified relevant cases from the PubMed and EMBASE databases using the search terms DOCK8 deficiency and acyclovir and DOCK8 deficiency and herpes. The eTable lists other reported cases of acyclovir-resistant HSV in DOCK8deficient patients. The majority of cases involved schoolaged females. Lesion types varied and included herpes labialis, eczema herpeticum, and blepharoconjunctivitis. Escalation of therapy and resolution of the lesion was seen in some cases with administration of subcutaneous pegylated interferon alfa- $2 b$.

Treatment Alternatives-Acyclovir competitively inhibits viral DNA polymerase by incorporating into elongating viral DNA strands and halting chain synthesis. Acyclovir requires triphosphorylation for activation, and viral thymidine kinase is responsible for the first phosphorylation event. Ninety-five percent of cases of acyclovir resistance are secondary to mutations in viral thymidine kinase. Foscarnet also inhibits viral DNA polymerase but does so directly without the need to be phosphorylated first. ${ }^{6}$ For this reason, foscarnet often is the drug of choice in the treatment of acyclovir-resistant HSV, as evidenced in our patient. However, foscarnet-resistant HSV strains may develop from mutations in the DNA polymerase gene.

Cidofovir is a nucleotide analogue that requires phosphorylation by host, as opposed to viral, kinases for antiviral activity. Intravenous and topical formulations of cidofovir have proven effective in the treatment of acyclovir- and foscarnet-resistant HSV lesions. ${ }^{6}$ Cidofovir also can be applied intralesionally, a method that provides targeted therapy and minimizes cidofovir-associated nephrotoxicity. ${ }^{12}$ Reports of systemic interferon alfa therapy for acyclovir-resistant HSV also exist. A study found IFN- $\alpha$ production by peripheral blood mononuclear cells in DOCK8-deficient individuals to be significantly reduced relative to controls $(P<.05))^{7}$ There has been complete resolution of acyclovir-resistant HSV lesions 
with subcutaneous pegylated interferon alfa- $2 \mathrm{~b}$ injections in several DOCK8-deficient patients. ${ }^{7-9}$

The need for escalating therapy in DOCK8-deficient individuals with acyclovir-resistant HSV infection underscores the essential role of DOCK8 in dermatologic immunity. Our case demonstrates that a high degree of suspicion for cutaneous HSV infection should be adopted in DOCK8-deficient patients of any age, regardless of acyclovir prophylaxis. Viral culture in addition to bacterial cultures should be performed early in patients with cutaneous erosions, and the threshold for HSV resistance testing should be low to minimize morbidity associated with these infections. Early resistance testing in our case could have prevented prolongation of infection and likely eliminated the need for a biopsy.

\section{Conclusion}

DOCK8 deficiency presents a unique challenge to dermatologists and other health care providers given the susceptibility of affected individuals to developing a reservoir of severe and potentially resistant viral cutaneous infections. Prophylactic acyclovir may not be sufficient for HSV suppression, even in the youngest of patients, and suspicion for resistance should be high to avoid delays in adequate treatment.

\section{REFERENCES}

1. Chu EY, Freeman AF, Jing $\mathrm{H}$, et al. Cutaneous manifestations of DOCK8 deficiency syndrome. Arch Dermatol. 2012;148:79-84. doi:10.1001/archdermatol.2011.262

2. Aydin SE, Kilic SS, Aytekin C, et al. DOCK8 deficiency: clinical and immunological phenotype and treatment options-a review of 136 patients. J Clin Immunol. 2015;35:189-198. doi:10.1007/s10875 -014-0126-0

3. Kearney CJ, Randall KL, Oliaro J. DOCK8 regulates signal transduction events to control immunity. Cell Mol Immunol. 2017;14:406-411. doi:10.1038/cmi.2017.9
4. Zhang Q, Dove CG, Hor JL, et al. DOCK8 regulates lymphocyte shape integrity for skin antiviral immunity. J Exp Med. 2014;211:2549-2566. doi:10.1084/jem.20141307

5. Engelhardt KR, Gertz EM, Keles S, et al. The extended clinical phenotype of 64 patients with DOCK8 deficiency. J Allergy Clin Immunol. 2015;136:402-412. doi:10.1016/j.jaci.2014.12.1945

6. Chilukuri S, Rosen T. Management of acyclovir-resistant herpes simplex virus. Dermatol Clin. 2003;21:311-320. doi:10.1016 /S0733-8635(02)00093-1

7. Keles S, Jabara HH, Reisli I, et al. Plasmacytoid dendritic cell depletion in DOCK8 deficiency: rescue of severe herpetic infections with interferon alpha-2b therapy. J Allergy Clin Immunol. 2014;133:1753 -1755.e3. doi:10.1016/j.jaci.2014.03.032

8. Papan C, Hagl B, Heinz V, et al Beneficial IFN- $\alpha$ treatment of tumorous herpes simplex blepharoconjunctivitis in dedicator of cytokinesis 8 deficiency. J Allergy Clin Immunol. 2014;133:1456-1458. doi:10.1016/j.jaci.2014.02.008

9. Metin A, Kanik-Yuksek S, Ozkaya-Parlakay A, et al. Giant herpes labialis in a child with DOCK8-deficient hyper-IgE syndrome. Pediatr Neonatol. 2016;57:79-80. doi:10.1016/j.pedneo.2015.04.011

10. Zhang Q, Davis JC, Lamborn IT, et al. Combined immunodeficiency associated with DOCK8 mutations. NEngl J Med. 2009;361:2046-2055. doi:10.1056/NEJMoa0905506

11. Lei JY, Wang Y, Jaffe ES, et al. Microcystic adnexal carcinoma associated with primary immunodeficiency, recurrent diffuse herpes simplex virus infection, and cutaneous T-cell lymphoma. Am J Dermatopathol. 2000;22:524-529. doi:10.1097/00000372-200012000-00008

12. Castelo-Soccio L, Bernardin R, Stern J, et al. Successful treatment of acyclovir-resistant herpes simplex virus with intralesional cidofovir. Arch Dermatol. 2010;146:124-126. doi:10.1001/archdermatol.2009.363

3. Shah NN, Freeman AF, Hickstein DD. Addendum to: haploidentical related donor hematopoietic stem cell transplantation for DOCK8 deficiency using post-transplantation cyclophosphamide. Biol Blood Marrow Transplant. 2019;25:E65-E67. doi:10.1016/j.bbmt.2018.11.014

14. Freeman AF, Yazigi N, Shah NN, et al. Tandem orthotopic living donor liver transplantation followed by same donor haploidentical hematopoietic stem cell transplantation for DOCK8 deficiency. Transplantation. 2019;103:2144-2149. doi:10.1097/TP.0000000000002649

15. Casto AM, Stout SC, Selvarangan R, et al. Evaluation of genotypic antiviral resistance testing as an alternative to phenotypic testing in a patient with DOCK8 deficiency and severe HSV-1 disease. J Infect Dis. 2020;221:2035-2042. doi:10.1093/infdis/jiaa020 


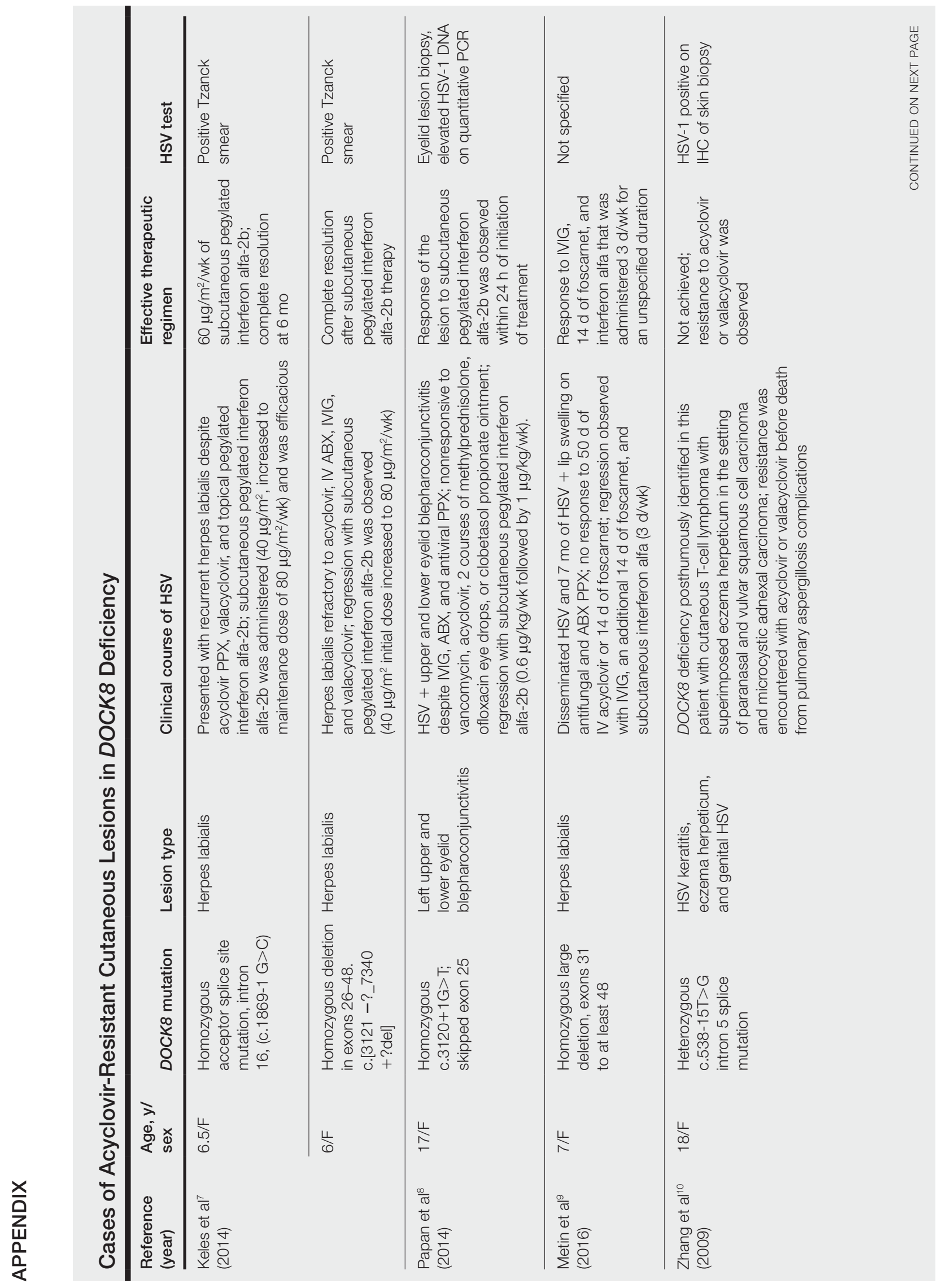




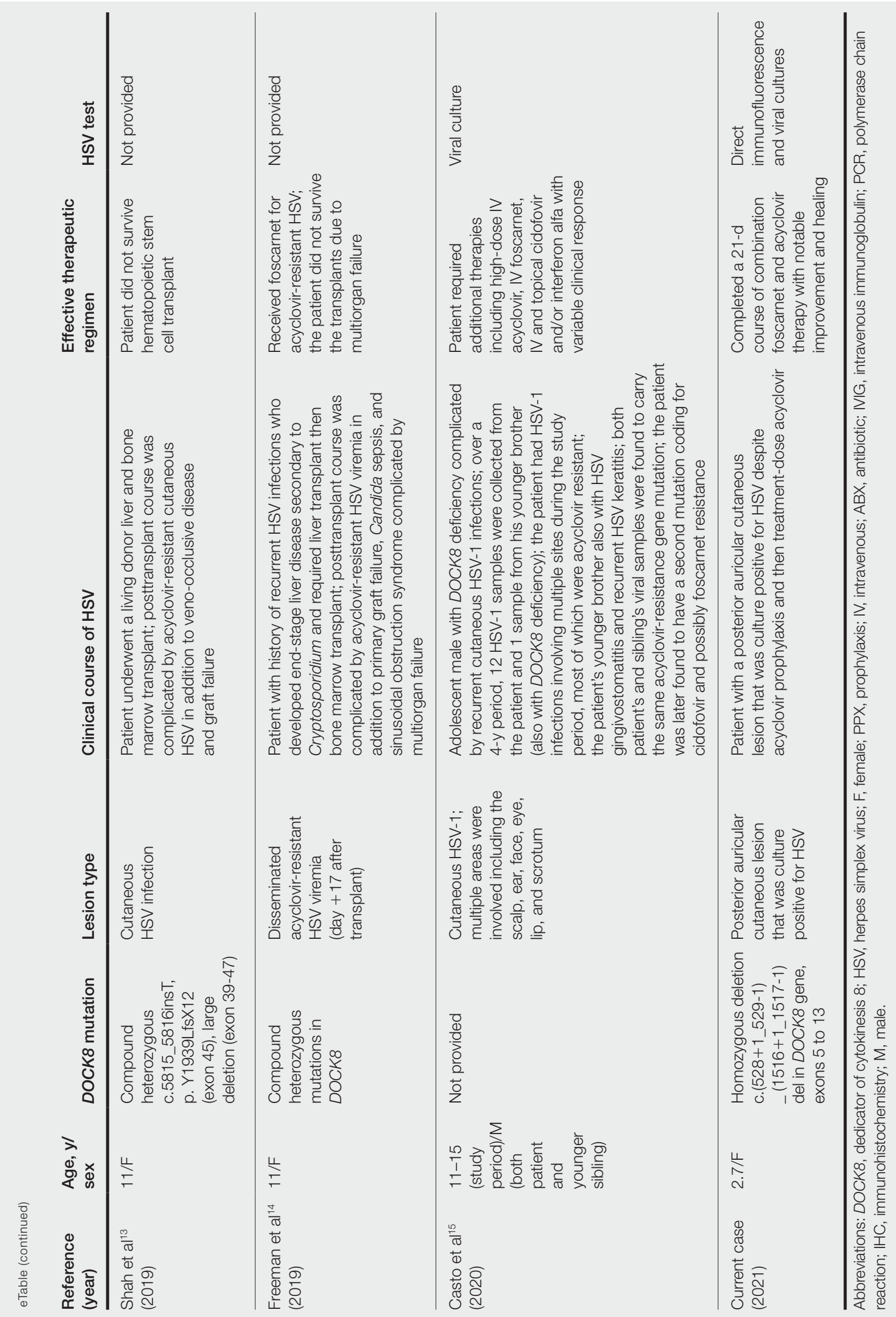

